\section{Zebrafish as an alternative method for toxicological studies}

\author{
Maria Sampieri, Riccardo Villa, \\ Silvia Dotti
}

Istituto Zooprofilattico Sperimentale della Lombardia e dell'Emilia Romagna, IZSLER; Centro di Referenza Nazionale per i Metodi Alternativi, Benessere e Cura degli Animali da Laboratorio, Brescia, Italy

\begin{abstract}
According to the Directive 2010/63/EU fish embryos do not fall into regulatory frameworks dealing with animal experimentation. Therefore, in compliance with the 3Rs principle, zebrafish embryos are considered as replacement or refinement methods. Since more and more industrial chemicals are recognized causes of skin sensitization, it is needed a thorough understanding of the mechanisms to make predictions of the toxic potential of novel compounds. Thus, the FET test was performed and up to four apical observations were recorded as indicators of lethality: coagulation of fertilized eggs, lack of somite formation, no detachment of the tail bud from the yolk sac and lack of heartbeat. Then, in order to assess whether the skin sensitization due to chemical incubation was really measurable, the Fish Interleukin 8 (IL8) ELISA Kit was carried out. The preliminary results obtained so far seem encouraging. However, they need to be confirmed through further ELISA tests and compared with other in vitro methods.
\end{abstract}

\section{Introduction}

The assessment of chemicals for potential toxic effects on both human health and the environment generates a strong demand for robust and cost-effective assays with high predictive power. Fish models are commonly used in human and ecotoxicological research to investigate the impact of chemicals on whole organisms. In fact, many important biological functions are conserved between fish species and humans. Zebrafish embryos are currently used as vertebrate models providing an intermediate step between in vitro and rodent assays as regards for example ecotoxicological studies. According to the
European Directive $2010 / 63$ and to the Italian Decree n. $2604 / 03 / 2014,{ }^{1}$ the National Reference Center for Alternative Methods, Welfare and Care of the Laboratory Animals in Brescia has been developing an in vitro method using zebrafish embryos to evaluate the toxicity of some compounds recognized as skin sensitizers. The study conducted consists of a first phase to evaluate the sensitizing potential of different chemical solutions through the FET test (OECD 236: 2013); ${ }^{2}$ which has been adapted to the needs of the aforementioned experimentation. This was followed by a second phase where Interleukin 8 (IL8) production was estimated, as there are studies showing that known skin sensitizers induce the production of proinflammatory cytokines and chemokines such as IL8. ${ }^{3}$ Once the sublethal concentrations of the tested compounds were identified, the production of IL8 was measured.

\section{Materials and Methods}

\section{Zebrafish mating and eggs production}

Fish selected for breeding were transferred to breeding tanks in the afternoon at a 2:1 M:F ratio. Zebrafish eggs were obtained from natural spawning of wild type animals in the early morning of the day after, following standard husbandry practices. After collecting embryos, these ones were placed in incubator at $28^{\circ} \mathrm{C}$.

\section{Dechorionation}

To remove the chorion, barrier that would hinder the passage of substances, at 24 hours post-fertilization (hpf) embryos were dechorionated using a protease isolated from Streptomyces griseus: Pronase. Embryos were transferred to Petri dishes filled with Embryo Medium 4 and incubated with Pronase diluted at a final concentration of $0,5 \mathrm{mg} / \mathrm{mL}$. After dechorionation, embryos were transferred to a new Petri dish with the test chemical solution for the FET test.

\section{Chemicals}

A careful and thorough review of the literature was necessary in order to identify the substances under investigation. The chemicals were selected for the availability of data on solubility (DMSO or saline) and on allergenic potency, such as 2,4Dinitrochlorobenzene (DNCB), Lactic acid (LA) and Sodium lauryl sulphate (SLS).

\section{Fish Embryo Toxicity Test}

The experimental design involves the incubation of embryos with the aforemen-
Correspondence: Maria Sampieri, Istituto Zooprofilattico Sperimentale della Lombardia e dell'Emilia Romagna, IZSLER; Centro di Referenza Nazionale per i Metodi Alternativi, Benessere e Cura degli Animali da Laboratorio, Brescia, Italy.

E-mail: maria.sampieri@izsler.it

Key words: Zebrafish; skin sensitization; ecotoxicology.

Conference presentation: this paper was presented at the Second Centro 3R Annual Meeting - 3Rs in Italian Universities, 2019, June 20-21, University of Genoa, Italy.

Received for publication: 28 October 2019. Accepted for publication: 11 November 2019.

This work is licensed under a Creative Commons Attribution NonCommercial 4.0 License (CC BY-NC 4.0).

CC Copyright: the Author(s), 2019

Licensee PAGEPress, Italy

Biomedical Science and Engineering 2019; 3(s3):118 doi:10.4081/bse.2019.118

tioned substances, the negative control represented by Embryo Medium and the solvent of the substances, of which a possible toxic effect must be excluded.

\section{Zebrafish exposure}

Dechorionated $24 \mathrm{hpf}$ zebrafish were exposed to the test chemical for a period of 96 hours. Every 24 hours, several observations were recorded: lack of heartbeat as indicator of lethality, while malformations such as a pronounced yolk sac oedema, a pericardial oedema or also a spinal curvature (scoliosis) were considered developmental toxicity endpoints. At the end of the exposure period, acute toxicity was determined based on any positive outcome in one of the four apical observations, then the $\mathrm{LC}_{50}$ was calculated.

\section{Tissue homogenization}

For this assay, 100 zebrafish larvae were collected in a $1.5 \mathrm{~mL}$ tube and homogenated: inside the Eppendorf tube was added a small bead and the instrument was setted for shaking at $30 \mathrm{~Hz}$ for $5 \mathrm{~min}$ utes. After that, the sample was centrifuged again for 10 minutes at $5000 \mathrm{~g}$, then the supernatant was removed and centrifuged for 30 minutes at $20000 \mathrm{~g}$.

\section{ELISA analysis}

Through the ELISA kit (performed according to the manufacturer's directions), the IL8 was evaluated. 


\section{ELISA IL8}

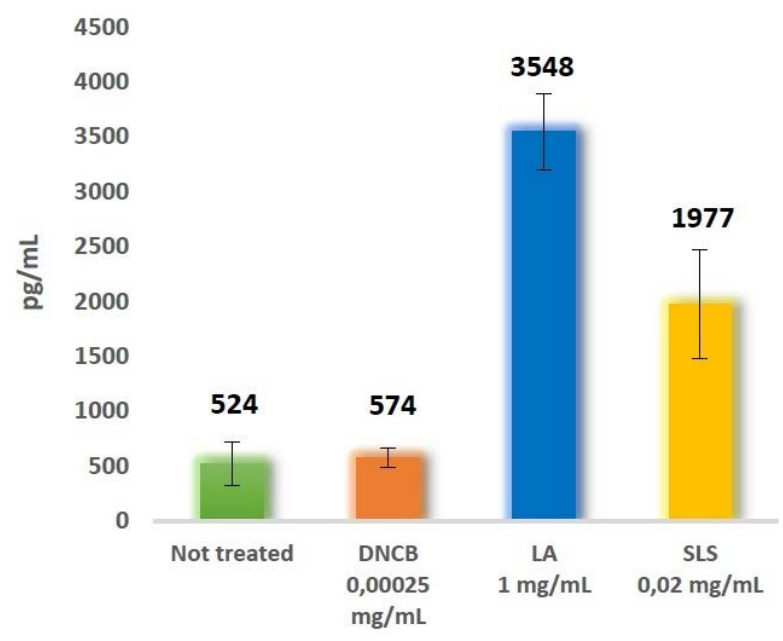

Figure 1. Quantitative evaluation of IL8 production after exposing zebrafish embryos at the highest concentration of DNCB, LA and SLS (embryos not treated were used as controls).

\section{Results}

From the $\mathrm{LC}_{50}$ values of each chemical agent, the substance that turned out to be the most toxic is the DNCB: $0,228 \mathrm{mg} / \mathrm{L}$ are indeed sufficient to determine the death of $50 \%$ of treated embryos. On the other hand, LA has proved to be the least toxic among those tested with an $\mathrm{LC}_{50}$ value of 1,172 $\mathrm{mg} / \mathrm{mL}$. As regards Sodium lauryl sulphate, its $\mathrm{LC}_{50}$ value observed was $0,041 \mathrm{mg} / \mathrm{mL}$.

Once the range of concentrations in which there is $100 \%$ survival was identified, IL8 production was measured. The results relating to the production of IL8 do not support, at the moment, a response in zebrafish larvae specifically correlated with the exposure to a sensitizing or non-sensitizing chemical. The quantitative evaluation of IL8 production was carried out by exposing the embryos for a period of 96 hours at the highest sublethal concentration of each compound (DNCB, LA and SLS) as shown in Figure 1.

From the preliminary results obtained, the FET test proved to be an excellent tool

\section{Conclusions}

to evaluate the toxicity of selected sensitizing substances and to analyze phenotypes that can be correlated with them. For this purpose, the test with chemicals already in use will be implemented and the range of chemical compounds to be evaluated will also be expanded. Moreover, this experimental model is extremely advantageous in the toxicological field thanks to the high fertility of the females: it was possible, indeed, to have a very large number of experimental units and to treat and analyze many embryos simultaneously. Further investigations are needed to clarify whether the mechanism of production of IL8 in zebrafish is correlated with the allergenic potency of a substance and also to obtain a complete framework to possibly widely apply this model in larger screening.

\section{References}

1. Legislative Decree n. 26 04/03/2014

2. OECD 236:2013. OECD Guidelines for the testing of chemicals. test n. 236: Fish embryo acute toxicity (FET) test. Paris, France: Organization for Economic Cooperation and Development; 2013.

3. Miyazawa M, Ito Y, Yoshida Y, et al. Phenotypic alterations and cytokine production in THP-1 cells in response to allergens. Toxicol In Vitro 2007;21:428-37.

4. Westerfield M. THE ZEBRAFISH BOOK, 5th Edition; A guide for the laboratory use of zebrafish (Danio rerio), Eugene, University of Oregon Press. Paperback, 2007. 